\title{
An IoT Based Efficient Waste Collection System with Smart Bins
}

\author{
Khandaker Foysal Haque*, Rifat Zabin ${ }^{\dagger}$, Kumar Yelamarthi*, Prasanth Yanambaka*, Ahmed Abdelgawad* \\ ${ }^{*}$ College of Science and Engineering, Central Michigan University, Mount Pleasant, MI, USA \\ ${ }^{\dagger}$ Chittagong University of Engineering and Technology, Dhaka, Bangladesh \\ Email: haque1k@cmich.edu
}

\begin{abstract}
Waste collection and management is an integrated part of both city and village life. Lack of optimized and efficient waste collection system vastly affect public health and costs more. The prevailing traditional waste collection system is neither optimized nor efficient. Internet of Things (IoT) has been playing a great role in making human life easier by making systems smart, adequate and self-sufficient. Thus, this paper proposes an IoT based efficient waste collection system with smart bins. It does real-time monitoring of the waste bins and determines which bins are to emptied in every cycle of waste collection. The system also presents an enhanced navigation system that shows the best route to collect wastes from the selected bins. Four waste bins are assumed in the city of Mount Pleasant, Michigan at random location. The proposed system decreases the travel distance by $30.76 \%$ on an average in the assumed scenario, compared to the traditional waste collection system. Thus it reduces the fuel cost and human labor making the system optimized and efficient by enabling real-time monitoring and enhanced navigation.
\end{abstract}

Index Terms-Internet of Things (IoT), Efficient Waste Collection, Optimized Route, Smart Bins.

\section{INTRODUCTION}

Internet of Things (IoT) is one of the fastest-growing technologies of the present world. IoT is a network of interconnected objects where these objects or things can transfer information among them without any direct interaction. It is inter-networking of the things involving sensors, actuators, embedded systems and many more which has many applications in industry, medical science, economy, weather forecasting, health monitoring and daily life [1] [2] [3]. IoT has led us to intelligent systems and devices which make our life a lot easier by making things efficient. The cities are becoming 'smart cities' by the virtue of IoT [4]. But waste collection systems are still a matter of concern. The waste collection system has not still improved rather remained inefficient. This waste collection system can be improved with the idea of IoT based smart bins and smart waste collection system.

Smart bin refers to a self-aware garbage collecting bin which can monitor the waste level of the bin by itself and thus decides when the wastes are to be collected. It also publishes the monitored data in the server and sends a notification to the system authorities. On the contrary, wastes are collected from the traditional bins at a regular interval irrespective of the necessity. Sometimes it might happen that, the bin is less than $50 \%$ filled and it is also not humid enough to facilitate the fast decomposition. In that case, the collection of the wastes would be unnecessary and inefficient. The smart bins let us know when the bins are filled or when the wastes are vulnerable to decomposition. It can make the waste collection system a way lot efficient and lessen human labor and cost. An embedded sensor network is necessary to convert the traditional waste bins to the smart ones. This embedded system consists of sensors that collect the necessary data like fill level, moisture level, temperature, etc. from the bins and are called IoT edge nodes.

The sensor data from the bins are sent to the cloud server. The transmitted data can be analyzed and represented in the most convenient way for an efficient waste collection system. The traditional waste collection system doesn't depend on the real-time data from the bins. Rather they collect the wastes in a particular time and particular path which is not always necessary. The monitored data from the smart bins can make life way easier. The collection system can wholly depend on the analysis of the monitored data to set the time and route for waste collecting. It not only reduces human labor but also decreases the fuel cost and maintenance cost by a great percentage. Fig 1 Shows an IoT based waste collection system with smart bins.

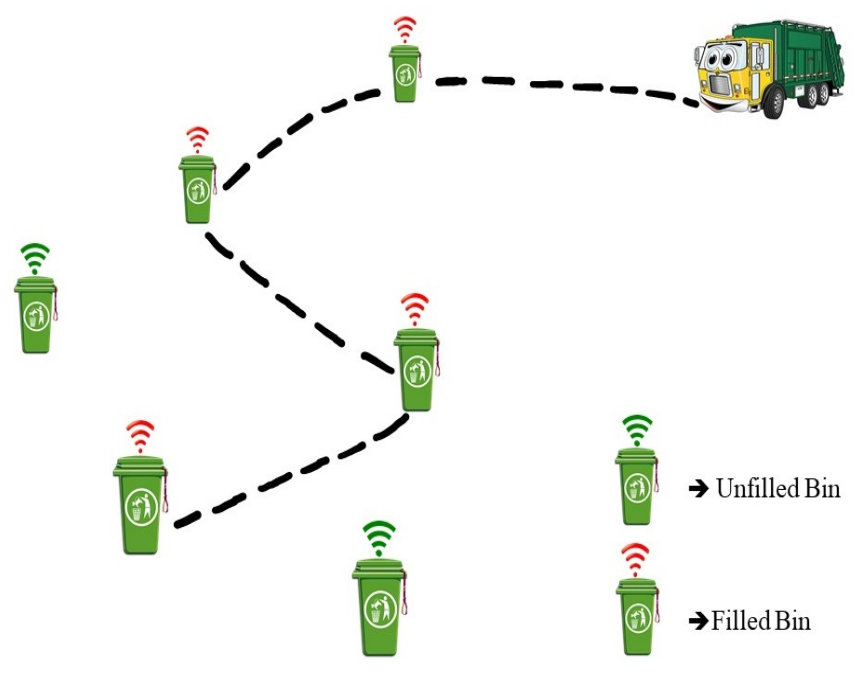

Fig. 1. A typical IoT based waste collection system

Here bins are equipped with IoT edge nodes which transfer the fill level to a cloud server. The waste collecting trucks 
collect the wastes from the filled bins only. This saves the fuel cost, human labor and time, decreasing the driving distance of the trucks. One of the major constraints of the embedded IoT edge nodes is the limited power source as they are battery-powered. It is troublesome and inefficient to change the batteries of the whole system now and then. So energy efficiency is one of the major concerns of the system. Another challenging issue is the navigation system for waste collection. The overall efficacy of the system depends upon it. Without an optimized navigation system, the waste collection process can not work most efficiently.

So we propose an IoT based waste collection system with an optimized navigation system which makes the waste collection much more efficient and saves both fuel and human labor. The rest of the paper describes the related works in this field, the proposed system design, results \& analysis and conclusion.

\section{RELATED WORKS}

In recent years IoT has grown forth in many folds. It has been creating many social relevant systems and applications which are making our life easier. Waste management and collection system are inseparable and important part of both city and suburb life. Thus it has already drawn the attention of the researchers. A lot of work is going on to improve the waste bins and waste collection system. Memon et al. proposed a smart garbage monitoring and collection system which can monitor the real-time garbage level [5]. This system is quite simple and straight-forward. WeMos D1 mini is used as the main processing unit of the system. This Arduino board is equipped with an HC-SR04 ultrasonic sensor which measures the waste level in the bin. When any client connects to the specified IP that the project board is assigned to, the system gets activated and starts collecting and publishing data. Otherwise, the system remains in sleep and saves energy. There is no notification scheme informing the garbage level in the proposed system. Moreover, it does not publish the data to any central server which can be monitored from anywhere any time. It also didn't show any waste collection procedure in the system. Adam et. al proposed a waste management system using IoT [6]. The bins are equipped with an embedded circuitry of ultrasonic sensor, Arduino Uno board and ESP 8266 module. Ultrasonic sensors measure the fill level of the bins which are then published to the server for monitoring using Transmission Control Protocol/Internet Protocol(TCP/IP). This system also shows the route to the bins from the base of the waste collecting trucks. Chen et al. propose an IoT system for waste management which uses the MQTT server for transferring the data from the bins to the database [7]. This system has also a provision for giving notification with a phone call when the sensor data exceeds a given threshold. It selects the waste bins based on the fill level by the infrared sensor and unpleasant smell by the gas sensor. This system has also used Google Maps to generate efficient routes for the truck to collect wastes. It uses LoRa for transferring the data from the bin to the gateway which usually has a wide coverage. But it is also constrained by the low data transfer rate. Susila et al. propose a smart waste collection and management system using IoT where the system sends a notification to the designated phone over GSM when the bin is full or left unattended for more than three days [8]. This allows the waste-collecting trucks to visit the required bins only which reduces the fuel cost and human labor to a great extent. But this has no routing system for collecting the wastes. Moreover, this system doesn't store the data in any cloud for real-time monitoring or to keep track of the data. Abdullah et. al propose an IoT based enhanced waste management architecture [9]. Here, every bin is connected to the cloud through GSM or GPRS and sends the data regarding, waste type, weight and volume. Bins send the information to the transportation system based on: (i) if the measured volume is more than that of defined threshold and (ii) if measured weight is more than that of defined weight. This method also enables different truck sizes depending on the amount and type of waste materials, which facilitate the traffic in densely populated cities. But waste volume and waste weight might not always be the accurate determinant of a filled up waste bin.

So, none of these systems provide a complete and structured solution for an effective waste collection process. The proposed system of this paper provides a thorough and efficient solution for the waste collection system with an optimized navigation system.

\section{SySTEM DESIGN}

The proposed waste collection system monitor all the waste bins of the defined area and update which bins need to be emptied in any cycle of collection of the waste. Moreover, it also has an efficient routing system showing the most optimized path for collecting wastes from the selected bins. It enables real-time monitoring of the waste level, odor and waste condition of the bins. The system can also monitor the temperature and humidity of the bins. Depending on these factors it can be decided which bins are ready for collection. The proposed system also shows the most optimized route which uses Google API. Fig. 2 depicts the whole proposed system. The waste bins of the system are equipped with an embedded system which acts as IoT edge nodes. These nodes collect the information from the bins and are capable of communication over $\mathrm{WiFi} / \mathrm{LTE} / 3 \mathrm{G}$. The collected data are stored in the IoT cloud and analyzed there. The data from the cloud are then published to the Thingspeak private channel as the user interface. Real-time data of the bins can be monitored any time from any remote location of the world. The bins data are utilized to detect, which waste bins are needed to be emptied as soon as possible and which ones can wait until the next cycle of waste collection. Depending on this information the proposed system optimizes the routes to collect the wastes from the bins most efficiently with minimum travel. The whole system architecture consists of mostly three sub-systems: (i) Smart bin system (ii) Real-time monitoring system and (iii) Navigation system. 


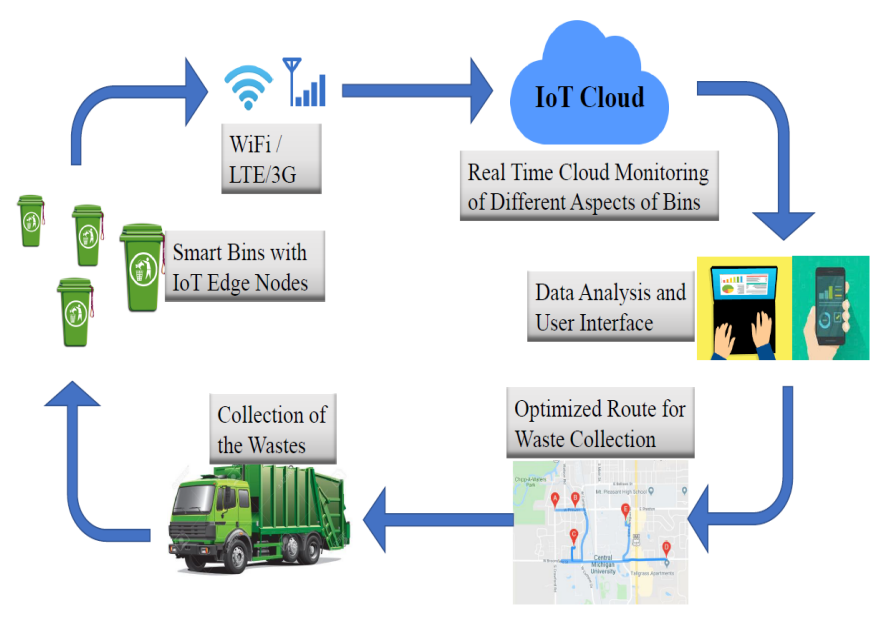

Fig. 2. The proposed system architecture

\section{A. Smart Bin System}

The Smart bins are capable of interacting with the surroundings to know its different aspects like fill percentage or fill level, odor level, humidity and let the central server know when it's ready for collection. For this purpose to serve, every bin of the system is equipped with an embedded system called IoT edge node. In every edge node, there are microcontrollers as computing units, communication transceivers, and sensors for collecting data from the bin. ESP8266 is used as the micro-controller unit which also supports IEEE 802.11 communication. The system incorporates BME 680 to measure the level of surrounding Volatile Organic Compounds(VOCs), temperature and humidity. VOCs in the waste bins are very hazardous which also spread bad odor. Moreover, VOCs may cause headaches including irritation in eyes, throat and nose. BME 680 measures the VOCs in kilo ohm as gas resistance values. IR sensor and Ultrasonic sensor (HC-SR04) in the node measures the fill status(filled or unfilled) and fill percentage respectively. ESP8266 collects all these data from the sensors and send them to the cloud over Wifi. The prototype of such smart bins has been implemented for the system. Fig. 3 shows the prototype of the smart bin which is equipped with the described IoT edge node. It continuously sends real-time data regarding fill status, fills percentage, VOC level, humidity and temperature data to the cloud.

\section{B. Real Time Monitoring System}

The real-time monitoring of the proposed system is done with the IoT cloud. The ESP8266 of the node is equipped with Wifi transceiver. After processing all the collected data from the sensors, the node sends them to the Thingspeak cloud. Real-time data are transferred to the cloud with an interval of 30 seconds. An interval of one hour for sending the data to the cloud significantly increases battery life. It is because the Wifi transceiver is power-hungry and the lifetime of the nodes increases with the increase of the data sending interval. The stored cloud data are presented to the user with the desired

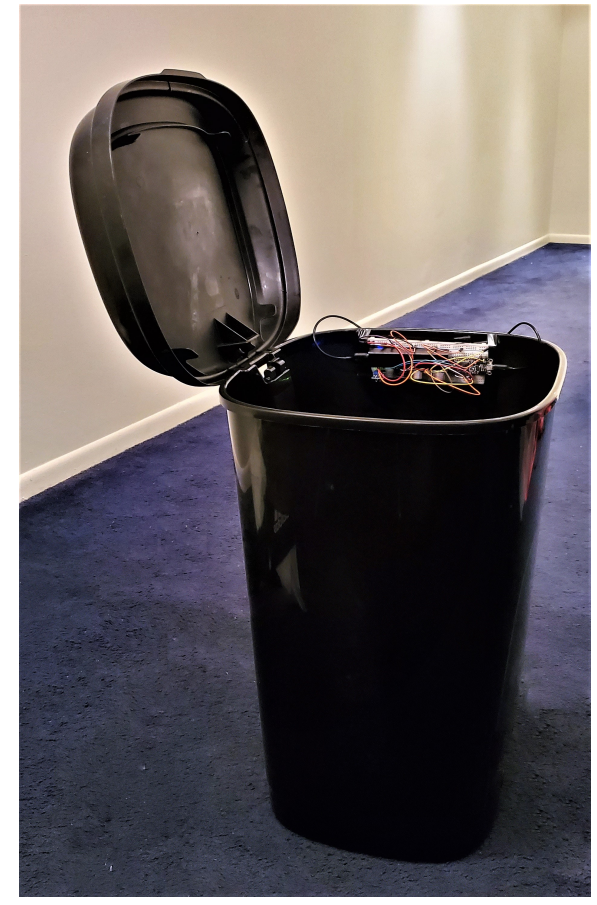

Fig. 3. Prototype of the smart bins

graphical interface. It also sends an email notification when the waste bin is filled $80 \%, 90 \%$ and $100 \%$. The system discards the random error by taking the mean of three values before sending the notification. Moreover, When the fill level reaches $100 \%$, the value of the IR sensor toggles from 1 to 0 which confirms $100 \%$ fill percentage.

\section{Navigation system}

The efficacy of a waste collection system highly depends on the navigation system to find the most optimized route among the bins which need to be emptied. The proposed navigation system is based on Google API and shows the most optimized driving route from source to destination with the way-points of selected bins. The waste collecting truck starts from its base and dumps all the collected wastes in another designated location and in its way it collects wastes from the selected filled bins. So, an optimized routing is needed to collect wastes only from the selected bins instead of all the bins in the area. For system design, four bins are considered in the random location of the city of Mount Pleasant, Michigan. For every cycle of waste collection, the user can choose one or multiple numbers of bins from the mentioned four bins depending on which are filled. Fig. 4 presents the route to collect wastes from waste bin 1(marker B on map) and waste bin 4(marker $\mathrm{C}$ on map). The truck starts from the truck base located at $\mathrm{A}$ and collects wastes from bin $1 \& 4$, depicted by marker B \& $\mathrm{C}$ respectively and goes to the destination located at $\mathrm{D}$.

Fig 5 shows the optimized route from base $A$ to the Destination D through the way-points of Waste bin 1 and 3 which are depicted by location marker B \& $\mathrm{C}$ respectively on 

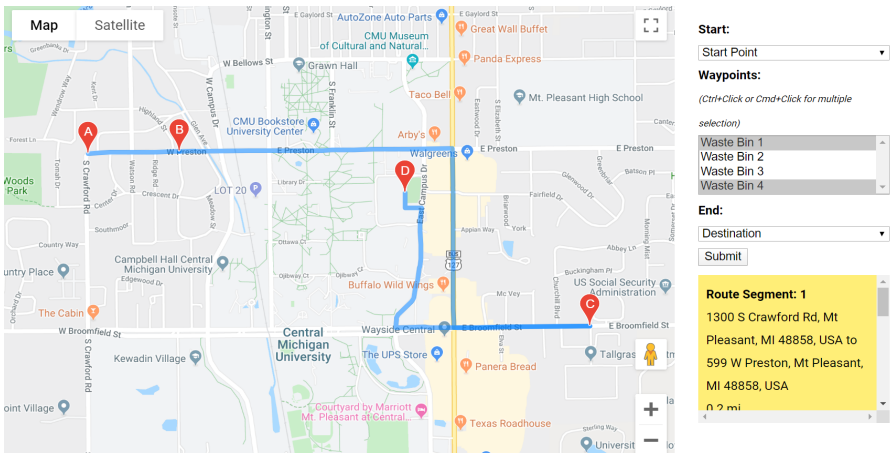

Fig. 4. Optimized route to collect waste from bin 1 and 4

the map. The navigation system provides all the names of the stops and also the distance of each route segment.
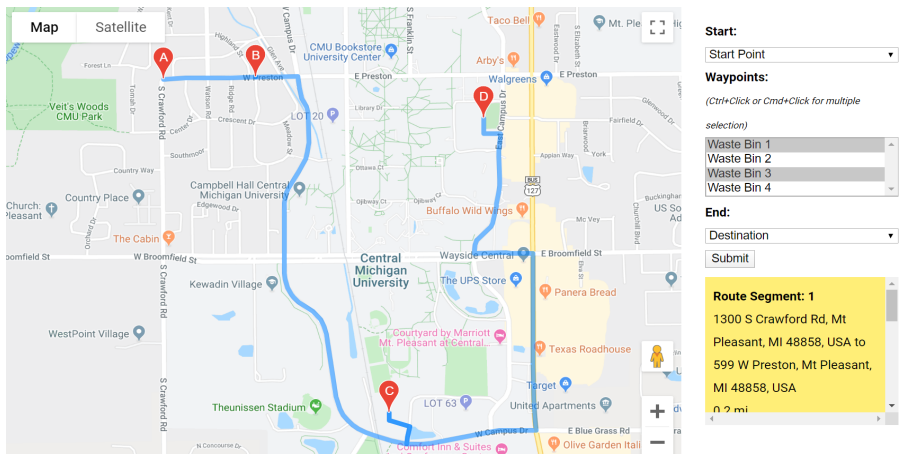

Fig. 5. Optimized route to collect waste from bin 1 and 3

\section{Results And AnAlysis}

Real-time data from the prototype smart bin is monitored. It gives fill percentage, fill status, VOC level, temperature and humidity of the bin. It is found to work as expected and give the corresponding data accurately. Fig. 6 presents only the fill level percentage over a period of 36 hours. It is showing the change of fill percentage as the bin gets filled up. It also sends email notification upon reaching the fill level $80 \%$ at $2 \mathrm{Pm}$, 25 December and $100 \%$ at 8 pm, 25 December.

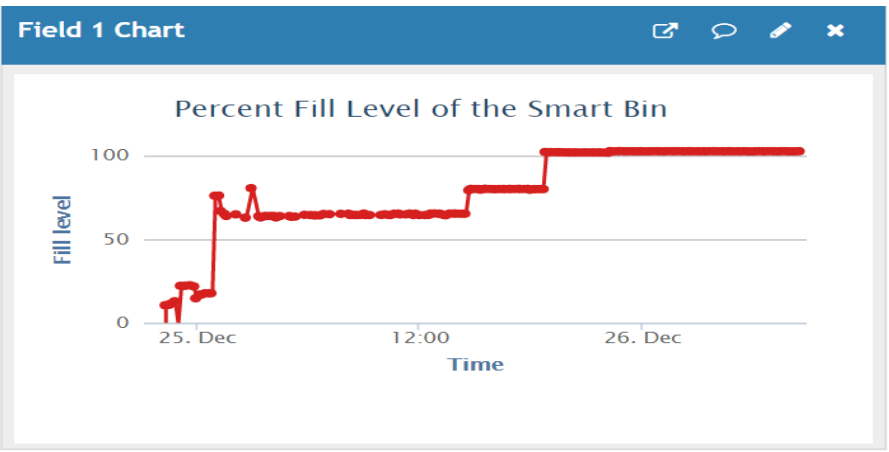

Fig. 6. Fill level percentage of the prototype smart bin

Fig 7 depicts the VOCs level in kilo-ohms over the time. The higher the gas resistance value better the air quality. In the indoor ambiance, fresh air gives gas resistance values of more than a hundred kilo-ohms.

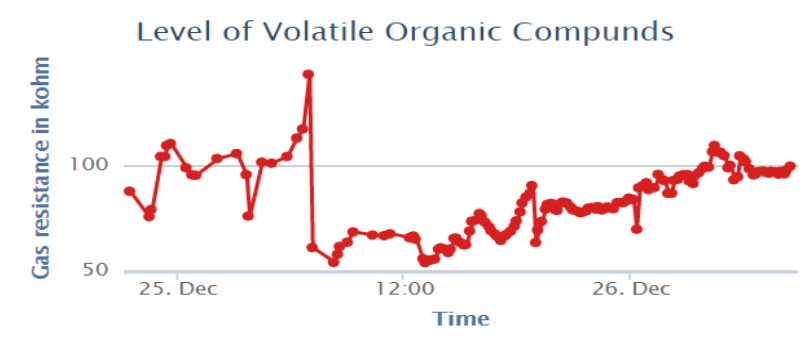

Fig. 7. VOC level of the prototype smart bin

At the beginning of the monitoring time, the gas resistance values remain in the range of normal air of 90-110 kilo-ohms. But with time the VOC level degrades with waste dumping which reaches to around 50 kilo-ohms at 2 pm, 25 December when the fill level reaches to $80 \%$. As the bin is kept open without cover, air diffusion improves the gas resistance values gradually. The proposed navigation system reduces the travel distance to a great extent and thus reduces human labor and fuel cost. The traditional system would collect wastes from all the bins irrespective of they are filled or not. If the truck starts from base A as described in fig. $4 \& 5$ and reaches to destination $\mathrm{D}$ after collecting wastes from all the four bins irrespective of their fill level, the driving distance would be 4.9 miles.

The proposed system makes the waste collection process efficient by decreasing the driving distance by a great percentage. Table 1. presents driving distances and percent decrease in the travel distance for different cases when compared to the

TABLE I

PERCENT DECREASE OF THE TRAVEL DISTANCE COMPARED TO THE TRADITIONAL WASTE COLLECTION SYSTEM

\begin{tabular}{|l|l|l|}
\hline $\begin{array}{l}\text { All the possible Cases with Pro- } \\
\text { posed System }\end{array}$ & $\begin{array}{l}\text { Travel Dis- } \\
\text { tance(miles) }\end{array}$ & $\begin{array}{l}\text { Decrease } \\
\text { of Travel } \\
\text { Distance in } \\
\text { Percentage }\end{array}$ \\
\hline Base-Bin1-Destination & 1 & $79.59 \%$ \\
\hline Base-Bin2-Destination & 2.8 & $42.86 \%$ \\
\hline Base-Bin3-Destination & 3.2 & $34.69 \%$ \\
\hline Base-Bin4-Destination & 2.9 & $40.82 \%$ \\
\hline Base-Bin1-Bin2-Destination & 2.7 & $44.90 \%$ \\
\hline Base-Bin1-Bin3-Destination & 3.3 & $32.65 \%$ \\
\hline Base-Bin1-Bin4-Destination & 2.8 & $42.86 \%$ \\
\hline Base-Bin2-Bin3-Destination & 4.2 & $14.28 \%$ \\
\hline Base-Bin2-Bin4-Destination & 3.8 & $22.45 \%$ \\
\hline Base-Bin3-Bin4-Destination & 4 & $18.37 \%$ \\
\hline Base-Bin1-Bin2-Bin3-Destination & 4.1 & $16.33 \%$ \\
\hline Base-Bin1-Bin2-Bin4-Destination & 3.7 & $24.49 \%$ \\
\hline Base-Bin1-Bin3-Bin4-Destination & 4.1 & $16.33 \%$ \\
\hline $\begin{array}{l}\text { Base-Bin1-Bin2-Bin3-Bin4- } \\
\text { Destination(traditional system } \\
\text { always chooses this route) }\end{array}$ & 4.9 & $0 \%$ \\
\hline
\end{tabular}


traditional system. It shows that the travel distance reduces significantly when the proposed architecture is followed instead of the traditional one. The average percent decrease in travel distance for the assumed scenario is $30.76 \%$. This makes the waste collection system optimized and efficient which saves both fuel cost and human labor. Table 2. compares the functionalities of the proposed system to other models. It clearly shows that, the proposed system has more functionalities and it is the most structured solution for efficient waste collection.

TABLE II

FunCTIONALITY COMPARISON OF DifFERENT MODELS

\begin{tabular}{|l|l|l|l|l|}
\hline Functionality & $\begin{array}{l}\text { Proposed } \\
\text { Model }\end{array}$ & $\begin{array}{l}\text { Smart } \\
\text { Garbage } \\
\text { Moni- } \\
\text { toring } \\
\text { System } \\
\text { by } \\
\text { Memon } \\
\text { et al. [5] }\end{array}$ & $\begin{array}{l}\text { Waste } \\
\text { Man- } \\
\text { agement } \\
\text { Model by } \\
\text { Chen et } \\
\text { al. [7] }\end{array}$ & $\begin{array}{l}\text { Smart } \\
\text { Waste } \\
\text { Col- } \\
\text { lection } \\
\text { System } \\
\text { by } \\
\text { Gutierrez } \\
\text { et al. } \\
{[10]}\end{array}$ \\
\hline $\begin{array}{l}\text { Fill Level Mea- } \\
\text { surement }\end{array}$ & $\checkmark$ & $\checkmark$ & $\checkmark$ & $\checkmark$ \\
\hline $\begin{array}{l}\text { Bad Odor Detec- } \\
\text { tion }\end{array}$ & $\checkmark$ & $x$ & $\checkmark$ & $x$ \\
\hline $\begin{array}{l}\text { Notification Sys- } \\
\text { tem }\end{array}$ & $\checkmark$ & $\checkmark$ & $\checkmark$ & $x$ \\
\hline $\begin{array}{l}\text { Error } \\
\text { Minimization for } \\
\text { Higher Accuracy }\end{array}$ & $\checkmark$ & $\boldsymbol{x}$ & $x$ & $\checkmark$ \\
\hline User Interface & $\checkmark$ & $\checkmark$ & $\checkmark$ & $\checkmark$ \\
\hline $\begin{array}{l}\text { Automated } \\
\text { Routing System } \\
\text { for } \\
\text { collecting Vehicle }\end{array}$ & & $\boldsymbol{x}$ & $\boldsymbol{x}$ & $\checkmark$ \\
\hline $\begin{array}{l}\text { Route } \\
\text { Optimization }\end{array}$ & $\checkmark$ & $\boldsymbol{x}$ & $\boldsymbol{x}$ & $\checkmark$ \\
\hline
\end{tabular}

The proposed system needs to be in the range of WiFi for communicating with the server which is one of the shortcomings of the system. To overcome this, we expect to implement this waste collection system with Routing Protocol for LowPower and Lossy Networks (RPL) which is an IPv6 routing protocol [11]. RPL will create a complete standalone system with Radio Frequency (RF) communication and this will also improve the network lifetime.

\section{CONCLUSION}

The proposed IoT based waste collection system does the real-time monitoring of smart waste bins. It allows a user to monitor fill level, fill status, VOC level, temperature and humidity of the waste bins from anywhere at any time. It also determines which waste bins are to be emptied at every cycle of the waste collection. Moreover, the navigation system presents optimized driving routes to collect wastes from the selected bins. The system decreases the travel distance significantly compared to the traditional system which eventually makes the waste collection process more efficient and also reduces the cost.

\section{REFERENCES}

[1] K. Yelamarthi, M. S. Aman, and A. Abdelgawad, "An applicationdriven modular iot architecture," Wireless Communications and Mobile Computing, vol. 2017, 2017.

[2] A. Abdelgawad and K. Yelamarthi, "Internet of things (iot) platform for structure health monitoring," Wireless Communications and Mobile Computing, vol. 2017, 2017.

[3] K. Yelmarthi, A. Abdelgawad, and A. Khattab, "An architectural framework for low-power iot applications," in 2016 28th International Conference on Microelectronics (ICM). IEEE, 2016, pp. 373-376.

[4] A. Caragliu, C. Del Bo, and P. Nijkamp, "Smart cities in europe," Journal of urban technology, vol. 18, no. 2, pp. 65-82, 2011.

[5] S. K. Memon, F. K. Shaikh, N. A. Mahoto, and A. A. Memon, "Iot based smart garbage monitoring \& collection system using wemos \& ultrasonic sensors," in 2019 2nd International Conference on Computing, Mathematics and Engineering Technologies (iCoMET). IEEE, 2019, pp. 1-6.

[6] M. Adam, M. E. Okasha, O. M. Tawfeeq, M. A. Margan, and B. Nasreldeen, "Waste management system using iot," in 2018 International Conference on Computer, Control, Electrical, and Electronics Engineering (ICCCEEE). IEEE, 2018, pp. 1-4.

[7] W.-E. Chen, Y.-H. Wang, P.-C. Huang, Y.-Y. Huang, and M.-Y. Tsai, "A smart iot system for waste management," in 2018 1st International Cognitive Cities Conference (IC3). IEEE, 2018, pp. 202-203.

[8] N. Susila, S. Anand, J. G. R. Elwin, and T. Sujatha, "Technology enabled smart waste collection and management system using iot," International Journal of Pure and Applied Mathematics, vol. 119, no. 12, pp. 12831295,2018

[9] N. Abdullah, O. A. Alwesabi, and R. Abdullah, "Iot-based smart waste management system in a smart city," in International Conference of Reliable Information and Communication Technology. Springer, 2018, pp. 364-371.

[10] J. M. Gutierrez, M. Jensen, M. Henius, and T. Riaz, "Smart waste collection system based on location intelligence," Procedia Computer Science, vol. 61, pp. 120-127, 2015.

[11] K. F. Haque, A. Abdelgawad, P. Yanambaka, and K. Yelamarthi, "An Energy-Efficient and Reliable RPL for IoT," in 2020 IEEE 6th World Forum on Internet of Things (WF-IoT). IEEE, 2020. 